\title{
Smoke-free legislation and prematurity
}

A recently publication (Cox B. et al. BMJ 2013;346:f441) shows the impact of avoiding exposure to tobacco smoke on pregnancy duration. It is impossible not to make certain considerations about the following when reading this publication: registries quality, laws implementation, the health system, and the importance of a population intervention regarding changes of individual behavior.

Flanders, in the North of Belgium, has 6 million inhabitants and 68 maternity centers where $99 \%$ of births take place. The quality of Flanders' registries is excellent and they record $99.8 \%$ of data regarding newborn infants in the region, the first link of any epidemiological study, fundamental for the development of health policies.

The 2011 Annual Report of the Ministry of Health of Argentina, published by the Statistics and Health Information Department, ${ }^{2}$ describes the work conducted to improve vital statistics data processing across the country. However, "problems related to data coverage and quality, are still present in some provinces. The magnitude of such omission is very important and has a negative impact on the validity of child mortality indicators."

"The government", "the country" or "the people" should not be blamed for our mistakes which are the product of our individual attitude when dealing with something for which we are irreplaceable: our daily duties. Illegible or incomplete medical records, birth or death certificates inaccurately filled in either by lack of knowledge or negligence, all contribute to the chronic lack of reliable data.

Preterm births, including births described as late preterm, constitute a burden of disease for the health system in the short-term (hospitalization in neonatal intensive care units), mediumterm (neurodevelopmental disorders, learning disorders), and long-term (irreversible sequelae and shorter life expectancy).

In addition, smoking is the main cause of preventable intrauterine growth restriction, and its harmful effects on mother and child health also include miscarriages, malformations, placental abruption, perinatal asphyxia, a higher risk of sudden infant death, asthma, atopy, etc.

According to the National Survey on Risk Factors, conducted in 2009 in Argentina, 26\% of women of childbearing age smoke (average:
9.4 cigarettes / day) and 34\% of them are exposed to second-hand smoke. In Argentina, the percentage of pregnant women who smoke (15$20 \%$ ) is one of the highest in the Americas and the world. ${ }^{3}$

Active and passive smoking during pregnancy causes low birth weight and preterm birth, among other harmful effects. All health care providers involved in the care of pregnant women are recommended to make a brief intervention towards smoking cessation at each antenatal care visit because it could be beneficial at any time during pregnancy.

Individual cessation strategies (brief intervention of health care providers) can be complemented with community strategies (advertisement regulation, smoke-free environments), thus strengthening their efficacy.

Results can be measured as an impact on individual health or as epidemiological indicators, such as smoking prevalence, exposure to second-hand smoke, or incidence of diseases directly related to smoking. In Scotland, the implementation of smoke-free environment laws led to a reduction in hospitalizations due to childhood asthma (Mackay D. et al. N Enl J Med 2010;363:1139-45)

From an individual standpoint, only a few months are required to experience the benefits of smoking cessation on lung function and circulation. But five to fifteen years are required to have a reduction in the risk of stroke, coronary heart disease and cancer. ${ }^{5}$

In the study mentioned before, Cox et al. selected the pregnancy cycle, only 9 months long, to measure the impact of smoking cessation. The placenta is responsible for providing the energy necessary for fetal growth. In addition to its endocrinological functions, the placenta is basically an organ responsible for the circulation of nutrition and gas exchange. The placenta lives nine months, matures and declines at the end of pregnancy, or earlier if normal functioning is affected. All causes of circulation alterations affect the placenta and the developing fetus, and tobacco is one of the most harmful factors.

Researchers found a reduction in preterm births with all the stages of implementation of smoke-free laws and a continuous descending trend in the years following the last stage.

No reduction was found in the rate of children 
with a birth weight of less than $2500 \mathrm{~g}$ or small for their gestational age, but it should be noted that induced labor deliveries were excluded from the analysis, which means that cases of intrauterine growth restriction leading to pregnancy termination might not have been considered.

One of the limitations of this study is that it did not take into account other factors that may modify preterm birth incidence or the number of antenatal controls and maternal conditions (hypertension, diabetes, drug use).

As pointed out by the authors, the beneficial effect of smoke-free legislation may arise from the non-exposure of pregnant women to tobacco smoke, but may also be due to the pregnant woman's and her family members' awareness regarding the dangers of tobacco.

Current smoke-free laws in Argentina are part of the World Health Organization Framework Convention on Tobacco Control.

In 2003, the 192 Member States of the World Health Organization (WHO) unanimously adopted this Convention, the world's first public health treaty, a legal instrument designed to reduce tobacco-related deaths and disease around the world.

Also in 2003, the Ministry of Health of Argentina started the National Program for Tobacco Control. One of the measures taken towards the program objectives was to pass and implement laws aimed at creating smoke-free districts because they constitute the basic unit of community life where actions are needed in relation to the reduction of smoke-free exposure.

It is worth noting the progress of legislation at provincial and municipal level since the creation of the National Program on Tobacco Control to 2010. A partial (55\% of population) or total (35\% of population) protection against tobacco smoke has been introduced in 18 of the 23 Argentine provinces and in the Autonomous City of Buenos Aires. ${ }^{6}$

In June 2011, a tobacco control law (National Law 26687) was passed, which established that "smoking is banned in all enclosed public or private spaces, including casinos and bingos, night clubs, bars and restaurants, theaters, museums, libraries, public transportation, and indoor stadiums. It is expressly prohibited to smoke in workplaces, either private or public. This prohibition will not apply to backyards, terraces, balconies and other open spaces for public access, except for schools and health facilities, where smoking will not be allowed including backyards."

Argentina is a federal country, and Article 39 of Law 26,687 invites "all provinces and the Autonomous City of Buenos Aires to pass regulations similar to those established by this Law at a national level applicable within their exclusive jurisdiction." ${ }^{7}$

Smoke-free legislation favors the overall population and also helps smokers individually in their smoking cessation attempts, modifies social behavior regarding the acceptance of tobacco consumption, and reduces public health expenditure related to associated diseases.

This is not only a matter of prohibiting tobacco consumption because it is also necessary to provide smokers with a chance to get away from tobacco.

There certainly are some sectors sustained by the marketing of tobacco-derived products that result financially harmed, but at the same time smoke-free environments are supported by irrefutable scientific evidence and an increasing social consensus

Norma Rossato, M.D. Associated Editor

http:/ /dx.doi.org/10.5546/aap.2013.186 